\title{
Anticiper l'activité par les configurations d'usage : proposition méthodologique pour conduite de projet
}

Anticipating activity through settings of usage: methodological proposal for project management

\section{Francisco Duarte et Francisco Lima}

\section{OpenEdition}

\section{Journals}

Édition électronique

URL : http://journals.openedition.org/activites/314

DOI : 10.4000/activites.314

ISSN : $1765-2723$

Éditeur

ARPACT - Association Recherches et Pratiques sur les ACTivités

\section{Référence électronique}

Francisco Duarte et Francisco Lima, «Anticiper l'activité par les configurations d'usage : proposition méthodologique pour conduite de projet », Activités [En ligne], 9-2 I Octobre 2012, mis en ligne le 15 octobre 2012, consulté le 30 avril 2019. URL : http://journals.openedition.org/activites/314 ; DOI : 10.4000 /activites. 314

\section{(c) $\oplus \Theta \Theta$}

Activités est mis à disposition selon les termes de la licence Creative Commons Attribution - Pas d'Utilisation Commerciale - Pas de Modification 4.0 International. 


\title{
Anticiper l'activité par les configurations d'usage : proposition méthodologique pour conduite de projet
}

\author{
Francisco Duarte
}

Université Fédérale de Rio de Janeiro, BP 68507 CEP 21945.970 Rio de Janeiro/RJ, Brésil - duarte@ pep.ufrj.br

\section{Francisco Lima}

Université Fédérale de Minas Gerais, Belo Horizonte/MG, Brésil - fpalima@ufmg.br

\begin{abstract}
Anticipating activity through settings of usage: methodological proposal for project management. The intervention of ergonomists in the early stages of the design process depends on the ability to predict the future activity characteristics of transformational work situations. The paradox of ergonomic design has been addressed using several different approaches. Typical situations of action, practical schemes and transformational models based on certain courses of action provide designers with information on the use of technical systems. These concepts deal with the transition between analysis of work in actual situations and the need to guide the design process. To this same end, in this paper we introduce the concept of settings of usage. We present examples of this concept to guide the design of future oil platforms beginning with the initial baseline studies. Settings of usage are intended to capture experiences gained in the operation of platforms and to transfer the accumulated knowledge to future projects, creating appropriate working conditions for the activity. The goal is to create, as a complement to the expertise of the operators, ergonomists and designers, a knowledge base organized around the settings of usage. This will enable the transfer of experiential knowledge before the project teams have even been constituted.
\end{abstract}

\section{KEYWORDS}

experience transfer, activity centered design, ergonomics guidelines, setting of usage

\section{1.- Introduction : ergonomie et modèles de l'activité pour la conception}

L'ergonomie contemporaine est née avec la prétention de proposer aux ingénieurs des recommandations afin d'incorporer le facteur humain au sein des projets technologiques en alliant enjeux de production et de santé. Ce problème de fond est toujours d'actualité et se pose encore dans les nouveaux projets et lors de l'implantation de nouvelles technologies. Depuis la fin des années 70, l'automation et la modernisation technologique de diverses unités de production industrielles ont fait l'objet de diverses études dans les perspectives socio-technique et ergonomique. Celles-ci mettent en évidence les dysfonctionnements et les problèmes significatifs liés à la gestion et à la conception de ces projets (Daniellou, 1988 ; Du Roy, Hunault, \& Tubiana, 1985). L'ergonomie a été ainsi sollicitée pour collaborer sous 
différentes formes avec les ingénieurs afin de leur fournir des orientations, principes, normes et paramètres ergonomiques et/ou pour travailler directement avec les équipes projet. Et très tôt, il y a eu une prise de conscience de la nécessité d'intervention en amont des projets qui définissent les situations de travail avant de prendre des décisions irréversibles ou qui alourdissent le coût des adaptations ergonomiques (Daniellou, 1988 ; Duarte, 2002).

L'efficacité de l'intervention ergonomique repose ainsi sur sa capacité à fournir à l'équipe de concepteurs, et ce dès le début du projet, des informations pertinentes sur l'activité des usagers. Le rapprochement entre l'ergonomie et l'ingénierie présuppose donc la production de connaissances qui soient applicables au projet avant la mise en place de certaines décisions. Ceci est réalisable si l'on fournit aux ingénieurs des connaissances sur le facteur humain, systématisées dans des manuels, des normes techniques et des guidelines et/ou par la collaboration directe de l'ergonome avec l'équipe du projet. Plusieurs problèmes se posent néanmoins pour rendre cette action effective, selon l'exemple du paradoxe de l'ergonomie de la conception (Theureau, \& Pinsky, 1984). En effet, pour transformer la situation de travail, l'ergonome doit connaître ses effets sur l'activité qui, à proprement dit, ne sera pas la même dans une situation future au regard de la complexité des éléments déterminants qui la configurent. "Mais si l'ergonomie attend qu'elle soit créée, il sera certainement trop tard pour réaliser des aménagements substantiels » (idem).

La «solution» de ce paradoxe passe par l'étude de l'activité de travail de situations similaires ou de situations de référence pour orienter le processus de conception. La connaissance de l'activité actuelle n'est pourtant que l'une des ressources qui contribuent à la construction de l'activité future. Selon Theureau et Jeffroy (1994), même lorsqu'il existe des connaissances sur l'activité, l'incorporation des recommandations ergonomiques dans les projets en développement n'est pas immédiate: "il y a un saut à faire, le saut de la connaissance de l'existant à la pratique de conception de ce qui n'existe pas encore» (Theureau, \& Jeffroy, 1994, p. 9).

Les connaissances traduites en recommandations et règles opérationnelles applicables par les concepteurs «projeteurs ${ }^{1}$ ne peuvent être communiquées en bloc et à un seul moment : à chaque étape du projet, il est nécessaire de fournir des informations plus ou moins détaillées, issues d'analyses correspondantes. L'analyse ergonomique doit avancer pas à pas avec le processus du projet. Pour contribuer à la conception des situations de travail, et ce, depuis les études initiales, cet article propose la notion de «configuration d'usage». Sur la base de l'analyse des situations existantes et des spécifications plus ou moins génériques du projet qui sont évaluées avec un regard critique au vu des observations de l'activité en situation de référence, des recommandations relativement abstraites sont élaborées. Ces dernières sont moins détaillées que les spécifications ergonomiques usuelles dans les études de détails des projets, mais elles sont toutefois plus effectives que les orientations génériques (comme «positionner la vanne dans un endroit facile d'accès ») qui ne servent que très peu aux concepteurs (Chapanis, 1996; Wulff, Westgaard, \& Rasmussen, 1999a et 1999b). La proposition de notion de configuration d'usage s'est inspirée, comme nous le verrons plus tard, de l'ouvrage A Pattern Langage de Christopher Alexander (1977).

Les configurations d'usage ont été élaborées en fonction de demandes de capitalisation de l'expérience des opérations de plateformes existantes pour de nouveaux projets dans un contexte décrit dans la seconde partie.

Face au constat de l'inefficacité des connaissances sur les «facteurs humains» pour influencer le projet, le retour d'expérience est ainsi apparu comme une alternative intéressante. C'est ce que nous présenterons en troisième partie de l'article.

1 On désigne sous le terme de projeteurs les agents en charge d'établir les projets dans les entreprises. 
Mais la notion de configuration d'usage rejoint des notions déjà connues de l'ergonomie de l'activité qui figurent dans la quatrième partie : les Situations d'Actions Caractéristiques (SAC) (Daniellou, 1992 ; Jeffroy, 1992), les schèmes d'utilisation et la genèse instrumentale du modèle SAI - Sujet, Activité et Instrument (Rabardel, 1995) ainsi que les propositions de transformation basées sur le Cours de l'Action - CA (Theureau, 2004 ; Theureau \& Jeffroy, 1994).

Après avoir défini minutieusement la notion de configuration d'usage, des exemples sont fournis pour orienter les projets de plateforme de pétrole ainsi que l'approche méthodologique développée pour leur élaboration dans une cinquième partie. Dans la conclusion, nous reprenons cette notion en discutant les points communs et les différences entre les différentes approches (SAC, SAI et CA).

\section{2.- Introduction de l'ergonomie dans les projets offshore}

La connaissance de la réalité du travail dans des situations de référence est une dimension stratégique pour le succès des projets d'ingénierie, car elle permet au futur corps technique de l'opération d'anticiper les problèmes auxquels il pourra faire face. Éviter les difficultés opérationnelles doit permettre aux nouvelles unités de production de réaliser une entrée en fonctionnement aux dates prévues, et ce, en mode stabilisé. Cela leur assure aussi de réaliser la production en termes de qualité et de quantité.

Mais cette action anticipée depuis les phases initiales du projet se voit confrontée aux modifications de la gestion de projet actuellement en cours dans l'industrie pétrolière brésilienne. Afin de mieux saisir ces modifications, rappelons qu'un projet industriel se divise couramment en cinq grandes phases. Chacune avec des démarrages, des contingences, des acteurs et des sorties propres (voir Tableau 1).

Actuellement, dans les entreprises de production de pétrole, le projet détaillé est sous-traité à des entreprises d'ingénierie spécialisées en gros projets industriels. Au fur et à mesure de l'accroissement des exigences de contrôle dans les projets, on peut observer une transformation dans la conduite de ces derniers. Avant, celle-ci était verticalisée et assumée en interne par l'entreprise de production de pétrole. À présent, elle est organisée en partenariats complexes et en réseaux à l'échelle mondiale. Avec ce phénomène de soustraitance, on perd néanmoins le contrôle du résultat final puisqu'une étude de base peut conduire vers différentes solutions en termes de coût et de qualité. Pour résoudre ce paradoxe - déléguer la conduite du projet, mais maintenir le contrôle sur la performance du produit final - les entreprises utilisent différentes stratégies : la définition précise de critères et de spécifications auxquelles le projet doit correspondre, voire même des contrats du type turn key ou production key.

Dans ce cadre, diverses normes et spécifications ergonomiques surgissent également, ou plus spécifiquement de facteurs humains, à appliquer dans les projets. L'hypothèse sous-jacente de cet effort de production de normes, réalisé internationalement par divers organismes publics, privés ou par les propres entreprises, consiste à fournir aux ingénieurs des spécifications ergonomiques pour aboutir à des projets d'installations plus efficaces, avec des conditions de travail plus adéquates. À cet effet, la construction de normes est la réponse dominante pour prévenir, par exemple, les problèmes ergonomiques dans la manipulation des vannes (Attwood, Deeb, \& Danz Reece, 2004 ; Chengalur, Rodgers, \& Bernard, 2004) et réduire les risques d'accidents. 


\begin{tabular}{|c|c|}
\hline Projet conceptuel & $\begin{array}{l}\text { Dans cette étape, les directives à suivre dans le projet actuel sont créées à partir } \\
\text { des orientations générales des projets de l'entreprise. } \\
\text { Acteurs principaux: Techniciens des domaines de l'Ingénierie et de } \\
\text { l'Exploration et de la Production }\end{array}$ \\
\hline Projet de base & $\begin{array}{l}\text { À partir des directives élaborées dans la phase préalable, divers documents sont } \\
\text { créés (fluxogrammes de processus, spécifications techniques et cahiers de } \\
\text { paramètres d'équipements, rapport descriptif) et des plans (vision générale) pour } \\
\text { les diverses zones de l'installation. Durant cette phase, des évaluations de risque } \\
\text { sont déjà réalisées (selon les techniques de HAZOP - HAZard and OPerability } \\
\text { study). } \\
\text { Acteurs engagés: Techniciens du domaine de l'ingénierie de base de l'entreprise }\end{array}$ \\
\hline Projet détaillé & $\begin{array}{l}\text { Les projets sont développés à un degré de précision qui permet leur construction. } \\
\text { On constate une forte division du travail entre les différentes spécialités } \\
\text { d'ingénieurs et le besoin de compatibilité entre les différents projets en cours. } \\
\text { Dans cette étape, la maquette électronique est conçue et des réunions de design } \\
\text { review se tiennent qui comptent sur la participation de techniciens de différentes } \\
\text { spécialités et de représentants de la future équipe opérationnelle. } \\
\text { Acteurs engagés: augmentation considérable du nombre de techniciens et } \\
\text { d'ingénieurs concernés, tout comme de représentants des futures équipes } \\
\text { d'opération et de maintenance (futurs usagers). }\end{array}$ \\
\hline $\begin{array}{l}\text { Exécution ou } \\
\text { construction }\end{array}$ & $\begin{array}{l}\text { Cette étape a lieu dans les chantiers navals et demande des ajustements et des re- } \\
\text { conceptions des projets développés durant les phases antérieures. Il s'agit d'une } \\
\text { étape dans laquelle diverses décisions sont prises, spécialement pour le projet de } \\
\text { tubulures et d'installations externes qui ont un fort impact sur les conditions de } \\
\text { travail des futurs opérateurs. Plusieurs entreprises de différentes spécialités } \\
\text { travaillent sur le même lieu, ce qui demande des efforts de coordination. } \\
\text { Acteurs engagés: techniciens de chaudière, de soudage, de peinture, monteurs } \\
\text { d'échafaudages, en plus de techniciens d'autres spécialités. }\end{array}$ \\
\hline $\begin{array}{l}\text { Commissionnement } \\
\text { et démarrage }\end{array}$ & $\begin{array}{l}\text { Les équipements sont testés par des essais qui rassemblent la participation des } \\
\text { futurs usagers et des représentants des fournisseurs. } \\
\text { Acteurs engagés : équipes de production et de maintenance des installations et } \\
\text { techniciens des différents fournisseurs d'équipements. }\end{array}$ \\
\hline
\end{tabular}

Tableau 1 : Étapes du projet et principales caractéristiques

\section{Table 1: Stages of the project and main characteristics}

La notion de configuration d'usage développée dans cet article est le résultat d'un projet réalisé en partenariat avec une grande entreprise brésilienne qui sous-traite le développement des projets et la fabrication de ses installations industrielles, mais qui cherche néanmoins à maintenir le contrôle sur la définition du projet de base en visant à garantir la sécurité, la performance et la fiabilité de ses unités de production. Garantir ces objectifs dépend encore du perfectionnement de la gestion des projets, et particulièrement de la capitalisation de l'expérience des projets antérieurs. Bien que les équipes internes d'ingénierie dominent toujours plus les technologies spécifiques de développement de projets d'unités de production, telles que les plateformes d'exploration en eaux profondes et ultra-profondes, le transfert d'expérience entre les projets est difficile au regard de certains aspects caractéristiques actuelles de l'entreprise :

1. l'accroissement rapide et concentré sur les dernières années de la demande de projets de nouvelles unités ;

2. la forte diminution du temps dédié au développement de ces projets lié à la quantité de projets et à la stratégie d'auto-suffisance nationale de production de pétrole ;

3. la grande quantité d'équipes engagées dans le projet et la diversité des sollicitations respectives ; 
4. l'augmentation croissante du coût des projets, avec en parallèle la sous-traitance de la gestion et du développement des projets, de la construction, voire même de l'opération des unités de production ;

5. la singularité de chaque projet où les équipes de projet, principalement celle de la coordination, sont toujours formées par des groupes différents, ce qui limite l'accumulation d'expérience ;

6. le manque d'analyses systématiques des unités en opération par les équipes de projet.

Un contexte semblable est rapporté dans le cas des projets offshore en Norvège. Selon Pagenhart et Buser (1998), il y a eu une diminution significative du délai au cours des années 1990 par rapport aux années 70 et 80 . Cette évolution s'est accompagnée d'une réduction du coût opérationnel : les unités de production offshore projetées pour un POB - people on board - dont les effectifs étaient de 200 personnes sont passées à moins de 100 personnes durant cette période.

Répondre à ces demandes actuelles de projet, dans la perspective de l'ergonomie de l'activité, présuppose, entre autres, de favoriser le retour d'expérience de la production pour orienter les solutions d'ingénierie, renforçant ainsi le mouvement historique qui s'oppose à la séparation instituée au long du XX ${ }^{\text {ème }}$ siècle entre les sciences de la santé et les sciences de l'ingénierie ou, de façon plus générale, les sciences de l'artificiel (Simon, 1996). Mais, malgré l'effort des ergonomes et de la demande reconnue des concepteurs, les difficultés d'opérationnalisation du retour d'expérience sont profondément enracinées, ce qui explique pourquoi les paramètres ergonomiques et de santé en général ne figurent pas dans les projets d'ingénierie.

Les projets sont en effet encore techno-centrés (Rabardel, 1995). Et les décisions principales d'un projet de modernisation technologique ou de construction d'une nouvelle unité de production sont contrôlées de façon prédominante par les dimensions techniques et économiques. Selon les compétences des concepteurs et les contingences temporelles et financières auxquelles ils sont soumis, les questions liées à l'activité future des opérateurs quand elles sont abordées - sont i) reléguées au second plan par rapport à la dimension technique et ii) invoquées uniquement dans les phases finales du projet (Carbadella, 1997). Le travail est alors juste considéré comme une variable de régulation des insuffisances du projet technique et des dysfonctionnements du processus de production et non pas comme variable de l'action (Maline, 1994). C'est une tentative précaire pour réunir a posteriori ce qui fut dissocié au départ.

\section{3.- L'introduction de l'ergonomie dans les projets d'ingénierie par la voie des spécifications techniques}

Une première tentative de réintroduction des facteurs humains dans les projets d'ingénierie a été guidée par la définition de paramètres physiologiques et cognitifs applicables aux différents projets. Les connaissances sur le fonctionnement de l'homme et sa relation avec les objets, environnements et instruments de travail, accumulées par l'ergonomie, sont présentées dans des ouvrages connus (Chapanis, 1996; Granjean, 1998; Iida, 1990 ; Salvendy, 1982) dont la prétention est de servir de base pour les projets d'ingénierie. Les divers chapitres de ces manuels établissent des priorités et des paramètres à propos des relations entre fonctions humaines déterminées (la vision ou l'anthropométrie, par exemple) et certains aspects du milieu de travail (tels que les couleurs, l'éclairage, les espaces, les dimensions des postes de travail et les équipements). Pour chaque fonction humaine spécifique, il existe une définition des paramètres et des spécifications de confort et d'efficacité qui sélectionnent les aspects pertinents de l'ambiance de travail. Il est ainsi possible de définir l'intensité minimum de l'éclairage en fonction du type de tâche ou des zones de confort également en conformité avec certaines caractéristiques des tâches, telles 
que le poids des objets ou la précision des mouvements.

Des études spécifiques sur l'utilisation des normes ergonomiques présentent néanmoins les contraintes et les difficultés ressenties par les ingénieurs pour trouver des informations pertinentes au moment de la consultation des spécifications (Haslegrave, \& Holmes, 1994 ; Klein, \& Brezovic, 1986 ; Meister, \& Farr, 1967 ; Swierengga, Morton, \& Boff, 1999). Selon ces auteurs, la plus grande difficulté de l'utilisation des recommandations est liée au fait qu'elles ont très fréquemment une formulation très générale, c'est-à-dire non destinée à une application dans une situation spécifique de projet.

Mais il ne semble pas que le manque d'orientation pour une situation spécifique soit la cause principale de la non-utilisation des normes ergonomiques. Chapanis (1996), dans son livre Human factors in Systems Engineering, affirme que des centaines d'articles, de livres et de rapports techniques ont été écrits et comportent des conseils, suggestions et recommandations de facteurs humains pour les projets les plus divers. Selon lui, les ingénieurs, si tant soi peu qu'ils se procurent et lisent ces guidelines (qui souffrent du même défaut, leur généricité) considèrent que ces recommandations constituent surtout un fardeau supplémentaire dans l'ensemble des autres spécifications et problèmes techniques contenus dans les projets.

Ainsi, depuis le début des années 90, des spécifications ergonomiques sont fournies aux entreprises norvégiennes du secteur offshore (Wulff et al. 1999a et 1999b). Selon ces auteurs, la quantité de spécifications dans ce type de projet semble i) être en conflit avec la capacité de traitement des ingénieurs et ii) les confrontations avec les spécifications des autres domaines sont nombreuses; de nombreux concepteurs considèrent les spécifications ergonomiques ou de facteur humain exagérées ou très génériques; certains d'entre eux considérant encore que les spécifications ergonomiques retardent le projet et augmentent les coûts.

La littérature ergonomique semble toutefois reconnaître les limites des guidelines de la discipline, même lorsque l'on remplace les formules génériques par des guidelines spécifiques facilement compréhensibles et peu ambigües. Une alternative pour surmonter ces limites serait l'intégration des ergonomes et des représentants des futurs usagers aux équipes du projet (Hendrick, \& Kleiner 2002). Le manque de connaissances spécialisées en ergonomie et d'informations sur les besoins des différentes équipes d'opération et de maintenance serait surmonté par des méthodologies de projet participatives. La présence matérielle d'usagers au sein des équipes de projet serait ainsi suffisante pour traduire en temps réel, avec le soutien de l'ergonome, les besoins et les expériences des opérateurs.

Dans le domaine offshore spécifiquement, certains auteurs (Pagenhart, \& Buset, 1998) soulignent la nécessité de transfert pour les projets d'informations techniques de pratiques liées au milieu de travail, à l'efficacité et à la fonctionnalité des équipements issus des équipes opérationnelles. Selon ces auteurs, le cycle de vie des investissements dans ce domaine montre que le coût du transfert d'expérience, les approches participatives et particulièrement la communication ou le dialogue interpersonnel sont des méthodes évaluées plus positivement par les projeteurs alors que les rapports, les documents archivés, les bases de données et les normes sont évalués plus négativement (Pagenhart, \& Buset, 1998). Si la nécessité de recourir à des savoirs issus de la pratique opérationnelle est reconnue face à l'inefficience des normes, l'alternative semble reposer exclusivement sur une gestion de projet qui promeut l'intégration entre usagers et concepteurs, sans qu'il soit nécessaire de créer une médiation par la documentation de l'expérience accumulée. L'inefficacité des normes sans l'expérience est remplacée par la présence immédiate des usagers sans les normes.

Cette contre-position ne nous semble pas féconde et exige une voie intermédiaire. L'ergonomie de l'activité reconnaît également les difficultés de transfert de l'expérience de l'ici et maintenant pour les situations futures. Les méthodes et modèles intermédiaires, 
comme les Situations d'Actions Caractéristiques - SAC, les schèmes d'utilisation et les modèles de transformation basés sur le cours de l'action, visent à offrir aux concepteurs des informations sur l'utilisation des systèmes techniques. De la même façon, c'est ce hiatus entre la sauvegarde de l'expérience et sa récupération dans le cadre de projets d'ingénierie spécifiques que la notion de configuration d'usage prétend combler.

Les configurations d'usage ici proposées se situent donc à un niveau intermédiaire entre les guidelines en ergonomie et la participation directe de l'usager au projet. Plus que des propriétés du fonctionnement humain, elles reflètent et cristallisent l'expérience des opérateurs des différentes unités de production. Elles tentent de fournir tout au long du projet un support de différentes manières à la participation des usagers et de l'ergonomiste. Durant les étapes du projet de base, les configurations d'usage sont suffisantes pour orienter les décisions générales, qui seront détaillées par la suite dans les projets d'exécution et élargiront la marge de manœuvre de la future équipe du projet. Une fois l'équipe constituée, elles serviront alors pour identifier les situations de référence et les situations d'actions caractéristiques plus pertinentes du projet en cours, ceci en temps utile pour s'intégrer aux autres spécialistes concernés.

Nous vivons une situation paradoxale. Tandis qu'il y a une reconnaissance de la nécessité de récupérer l'expérience des travailleurs, des inadéquations ont encore lieu au sein de nouveaux projets. Inadéquations liées à la non-incorporation du savoir accumulé en opération et en maintenance des systèmes productifs. Cette perte d'expérience est encore plus intense et visible dans les milieux offshores, où les unités de production sont isolées et d'accès difficile pour les concepteurs. Cette méconnaissance est l'une des raisons da la sousestimation de la diversité des modes de fonctionnement des installations de production par les concepteurs et gestionnaires. Cela a été continûment mis en évidence par diverses études (Clot, 1998 ; Daniellou, 1988 ; De Keyser, De Cortis, \& Van Daele, 1988 ; Duarte, 1994 ; Laville \&Teiger, 1972 ; Ombredane, \& Faverge, 1955 ; Zarifian, 1995).

Ainsi, malgré la grande quantité d'études relatant la réalité des situations de travail, l'ergonomie semble souffrir du même problème de manque de retour d'expérience: les analyses de l'activité ont encore peu d'influence sur les projets de nouvelles installations. Il est évident que l'on ne peut attribuer aux ergonomes la responsabilité de la méconnaissance des situations de travail, ou aux analyses produites. En revanche, il faut identifier ces difficultés au sein du projet dont la rationalité est déterminée par de multiples facteurs: économiques, sociaux et politiques, qui échappent à l'action directe des ergonomes, voire des autres acteurs du projet. Toutefois, même quand il est possible d'agir, quand l'ergonomie s'est créé un espace et s'est intégrée dans la conduite du projet, différents problèmes se posent pour que l'expérience de la production puisse enrichir les solutions proposées. Quand il s'agit de soutenir un projet de conception, il est nécessaire de passer de l'analyse des situations existantes au projet des situations nouvelles. Ce qui est important n'est plus l'activité actuelle, mais celle qui pourra avoir lieu dans le futur. Mais quels sont les éléments de connaissance du travail et de ses variabilités à déplacer vers les processus de conception ? Comment le retour d'expérience est-il possible?

\section{4.- L'ergonomie de l'activité et le transfert d'expérience de la production vers le projet des nouvelles installations : modes d'anticipation et de participation}

En parallèle des études qui décrivaient la réalité des situations de travail, certaines propositions pour soutenir la conception ont été développées par l'ergonomie de l'activité depuis la fin des années 1970. Theureau et Jeffroy (1994) et Theureau (2004) proposent un «modèle de transformation », caractérisé comme un ensemble de représentations du cours des actions susceptibles de guider la conception de la situation future. Daniellou (1992), sur 
la base du concept de situations d'actions caractéristiques (SAC), a développé l'approche de la situation future possible. De la même façon, l'approche instrumentale se base sur le concept de schèmes d'utilisation pour soutenir le processus de conception (Béguin, 1994 ; Rabardel, 1995).

Les différentes propositions de l'ergonomie de l'activité pour intervenir sur le projet ont une caractéristique en commun : l'approche ascendante ou «bottom-up », d'autant plus effective quand elle peut interagir plus tôt avec les décideurs et les concepteurs (Daniellou, 1988 ; Maline, 1994). Le principe général est que la prise en compte des conditions de réalisation des activités de travail, depuis les étapes initiales d'un projet, peut orienter les choix concernant la conception globale des systèmes techniques et des postes de travail spécifiques, ce qui crée des systèmes techniques compatibles avec l'activité future de travail.

Ces approches de l'ergonomie de l'activité trouvent leur origine dans la notion de «modèle opérant » proposée par Wisner (1994) qui insistait sur l'infinie singularité des situations dans lesquelles l'ergonome agit. L'intervention devait ainsi partir d'un diagnostic et produire des modèles singuliers qui seraient les outils de transformation des situations spécifiques. Wisner (1972) appelait ces modèles, des modèles opérants et les définissait comme «des modèles de la situation qui soient représentatifs des aspects essentiels du réel, qui permettent des mesures objectives et qui sont susceptibles de conduire à des solutions efficaces». Un modèle est donc opérant quand il décrit les conséquences pour les opérateurs et quand il conduit à des solutions efficaces. Mais qu'est-ce qui est alors essentiel pour la construction du modèle opérant, si ce n'est ce qui définit la singularité de chaque situation ? Dans ce cas, quelle est la pertinence des modèles opérants pour faire face aux différentes situations dont ils sont issus ? Les propositions suivantes cherchent à répondre à ces questions à propos des relations entre situations singulières et modèles plus généraux de l'activité de travail.

\section{1.- L'approche de l'activité future}

Les demandes croissantes d'intervention sur les projets ont conduit au développement de l'approche de l'activité future autour de la question suivante: "peut-on construire des modèles scientifiques de l'activité de travail qui permettent de prévoir les conséquences des choix de projet en lien avec les domaines de l'efficacité et de la santé des opérateurs? (...) Si personne ne peut prévoir toutes les conséquences d'une série de choix, existe-t-il (...) un espace pour la définition d'un programme de recherche dont l'objectif serait d'améliorer la connaissance des conséquences prévisibles de tels choix sur l'activité de travail» (Daniellou, 2004, p. 191).

L'approche proposée par Daniellou (1992) se développe en deux temps : dans les situations de référence, il identifie et décrit des Situations d'Actions Caractéristiques - (SAC) avec plusieurs degrés de détails, descriptions combinées et structurées dans des scénarios qui instruisent des simulations de l'activité future probable/possible ${ }^{2}$. C'est à partir des réflexions sur l'activité future que s'établissent les négociations avec les concepteurs, dans le but non pas en vue d'obtenir un ajustement parfait et définitif entre les conditions de travail et l'activité, mais de créer un espace pour le développement de l'activité compatible avec la santé et les exigences de production. L'objet de la conception n'est alors pas l'activité, entité toujours énigmatique et relativement inappréhendable, mais l'espace dans lequel elle se

2 Si, à l'origine, l'approche proposée par Daniellou (1992) véhiculait la notion d' " activité future probable », cette notion fut remplacée par celle « l'activité future possible » en raison de l'impossibilité de prévoir l'activité future. Selon l'auteur, l'activité de travail serait une « construction originale d'un individu particulier dans une situation donnée, ce qui n'est pas prévisible dans sa singularité » (Daniellou, 2004, p. 191). L'approche de l'activité future possible ne serait donc pas une tentative de prévoir l'activité mais de prévoir des marges de manœuvre, de l'espace dans le cadre duquel l'activité pourra se déployer. 
déroule.

La SAC est alors l'unité élémentaire de l'ergonomie de conception (Maline 1994). Et ce concept (initialement développé par Jeffroy en 1987) est ce qui permet l'instruction du futur à partir de l'existant. Maline souligne le caractère opérationnel et l'utilité pratique du concept pour le processus de projet (Maline 1994, p. 80). Les SAC sont des caractéristiques de situations déterminées de travail actuelles qui permettent la construction du processus de simulation en ergonomie.

Les SAC élaborées par les ergonomes en interaction avec d'autres acteurs du projet à partir de situations de travail observées en situation de référence soulignent spécialement les formes de variations qui pourraient échapper aux concepteurs. Une SAC peut donc être plus ou moins spécifique. En c'est en fonction de la question prise en compte et de l'étape du projet que l'ergonome et les participants de la simulation choisiront le niveau de spécification le plus pertinent.

La description d'une SAC comporte au moins les éléments suivants: (i) les personnes concernées, (ii) les tâches à réaliser (i.e. Les objectifs à atteindre), les exigences (de temps, de qualité, de sécurité, de disponibilité de ressources de travail) et (iii) les facteurs de variation susceptibles d'influencer le déroulement de la situation (propriétés de la matièrepremière, jour/nuit, intempéries...) (Daniellou, 1992).

Cependant, la description et l'énumération des SAC susceptibles d'avoir lieu ou d'être utiles pour le projet fournissent une vision toujours partielle et fragmentée des conditions de réalisation du travail. Selon Maline (1994), dans le cadre du processus de simulation du travail, la phase suivante serait la construction de scénarios, c'est-à-dire l'enchaînement de SAC dans une perspective temporelle. Et ce sont les scénarios qui pourront enrichir la gestion du projet en faisant surgir les besoins des opérateurs en termes de fonctionnalités, de caractéristiques des équipements, mais également du point de vue de l'organisation du travail et de la formation. Les simulations, lorsqu'elles sont conduites dans le cadre d'une construction sociale d'importance, permettent de faire surgir les différences entre les représentations de chaque acteur et les interrelations entre les divers aspects du projet tout en favorisant l'intégration de l'ensemble (Béguin, \& Weill-Fassina, 1997 ; Daniellou, 1992 ; Garrigou, Daniellou, Carballeda, \& Ruaud, 1995).

Dans la reconstitution simulée de la situation de travail future, en plus des SAC, diverses données disponibles sur les espaces et les ressources du travail sont utilisées avec le support de plans et de maquettes (objets intermédiaires de la conception), d'informations sur la future population de travail et l'organisation du travail. Les SAC peuvent également être plus ou moins spécifiées mais leur degré d'abstraction est défini dans le processus propre de conception. La définition des SAC pertinentes pour le projet, ainsi que la construction et la formalisation des scénarios, sont réalisées selon une démarche participative. En d'autres termes, entre les SAC des situations de référence et les SAC futures, le travail de traduction est réalisé par l'intermédiaire de la construction sociale. Le processus de récupération de l'expérience des opérateurs a lieu ainsi dans l'interaction immédiate entre les acteurs durant le projet. Dans chaque nouveau projet, il faut reprendre le cycle de retour d'expérience, du fait du changement des acteurs : c'est ce que l'on retrouve également dans le cadre de grands projets d'ingénierie comme les plateformes de pétrole.

\section{2.- L'approche instrumentale et le modèle SAI}

Dans l'approche instrumentale, la notion de schème d'utilisation est la référence pour penser le neuf à partir de connaissances génériques issues d'expériences vécues. Son origine est associée à la notion de schème et à d'autres notions semblables telles que le schéma, le script et le scénario qui, bien qu'elles soient toutes construites à partir de différents cadres théoriques, proposent toutes la caractérisation des invariants qui structurent l'activité et l'action. La majorité des formalisations des notions de schème traite des relations entre les 
connaissances déclaratives et procédurales (Rabardel, 1995).

En présentant le point de vue de la psychologie sur l'instrument, comme une entité mixte (formée par un mécanisme et un schème d'utilisation), Rabardel (1995) expose plusieurs définitions de la notion de schème. Il débute avec l'étude de la définition de Piaget, pour qui le schème d'une action serait l'ensemble structuré de caractères généralisables de l'action qui permettent la répétition de l'action ou son application à de nouveaux contenus (Rabardel, 1995, p. 93). Ils représenteraient ainsi les moyens dont disposent les individus pour permettre l'assimilation de situations ou d'objets auxquels ils sont confrontés dans le quotidien.

La notion de «schème d'utilisation », c'est-à-dire les schèmes liés à l'utilisation d'un mécanisme et les différents niveaux de cette notion (schèmes d'usage, schèmes d'action instrumentée et schèmes d'activité collective instrumentée), comporte des dimensions privées et sociales. La dimension privée est propre à chaque individu alors que la dimension sociale est liée à la construction de schèmes en commun avec d'autres individus, d'autres usagers. Les concepteurs d'artefacts contribuent également à la construction des «schèmes d'utilisation ». Le caractère social des schèmes d'utilisation peut aussi être vu dans les mécanismes de transfert plus ou moins formalisés de ces derniers. Les exemples de transfert sont: (i) les informations transmises d'un usager à l'autre, (ii) les formations pour les opérateurs de systèmes techniques complexes et (iii) les divers types d'assistance à l'usager (manuels et modes d'utilisation variés incorporés ou non au propre artefact).

Pour Rabardel (1995), les schèmes d'utilisation «permettent d'attribuer des significations aux objets en fonction de l'orientation de l'activité du sujet et des tâches. Ils permettent de leur assigner des statuts d'une part en termes de buts et sous-buts, d'états, de changements d'états et transformations opérables sur les objets d'autre part, en termes de moyens, c'està-dire d'instruments pertinents pour des actions possibles ».

Les schèmes d'utilisation ne sont pas appliqués directement. Ce sont des spécifications en fonction du contexte et ils sont actualisés pour s'adapter aux singularités des situations. À la fois moyens pour faire face aux nouveaux objets et artefacts, ils peuvent également être considérés comme des schèmes familiers, facilement mobilisables pour contribuer au fonctionnement automatisé en situations habituelles. Dans une situation nouvelle, mais similaire toutefois, les schèmes d'utilisation conduisent à des généralisations par l'extension de classes de situations pertinentes. Ils peuvent néanmoins conduire à des différenciations quand il est nécessaire de s'accommoder et de s'adapter aux aspects effectivement différents des situations nouvelles.

Comme mentionnée, la notion de schème d'utilisation est à la base de la construction de l'approche instrumentale de la psychologie. Dans le cadre de cette approche, un artefact n'est jamais un instrument fini. Un artefact a besoin de s'insérer dans l'usage, c'est-à-dire dans les activités pour lesquelles il constitue un moyen pour atteindre des objectifs définis par l'usager. Cette utilisation excède pourtant l'anticipation des concepteurs, car selon une formulation de Béguin (1994), la conception se continue dans l'usage. Le développement des formes d'utilisation se prolonge ainsi au-delà de la conception initiale comme une production à la fois individuelle et collective. "Il s'agit de rendre compte de la production des usages par les utilisateurs, du développement de leurs compétences et d'élaborer une conceptualisation des processus de conception qui permette de comprendre cette contribution des utilisateurs à la conception et de faciliter sa prise en compte par les concepteurs professionnels $»$ (Béguin 1994, p. 95).

La position instrumentale de l'artefact est associée à son statut dans le cadre de l'action. Ce dernier n'est pas un instrument en soi ou une composante de l'instrument, il est institué comme instrument par le sujet qui lui donne le statut de moyen pour atteindre les objectifs de son action. De cette forme, les artefacts s'inscrivent au sein de l'activité et y provoquent des réorganisations plus ou moins importantes. Le même artefact peut ainsi avoir un statut 
instrumental très varié selon les sujets et pour le même sujet, selon les situations et les moments.

L'instrument, en tant que tel, et toutes ses composantes constituent ainsi une forme de capitalisation de l'expérience : une connaissance. Pour les sujets, un artefact s'enrichit de situations d'action où il a été inséré circonstanciellement et singulièrement en tant que moyen de son action. C'est ainsi que se constitue ce que l'on nomme la palette, le champ instrumental du mécanisme pour le sujet : l'ensemble des schèmes d'utilisation de l'artefact.

La contribution fondamentale de l'approche instrumentale réside dans le fait de reconnaître l'inscription des activités dans les artefacts en tant que dimension du processus de conception qui s'étend à l'usage. A proprement dit, le projet se complète seulement ou ne devient effectif que dans l'usage. Concevoir est une fonction continue qui s'identifie au développement de l'activité. Si cette approche permet une compréhension particulière du processus de conception comme dimension interne de l'activité, certaines questions d'ordre pratique subsistent : comment formaliser alors la variabilité de situations d'utilisation ou de schèmes d'utilisation des artefacts (palette) et les combinaisons entre les sujets, les actions et mécanismes (modèle SAI - Sujet/ - Activité/Instrument) ? Comment cette formalisation participe-t-elle à la conduite du projet de conception des artefacts ? Et à quel moment, avec quels contenus et niveaux de généralité ?

\section{3.- Modèles de transformation du cours de l'action}

L'approche du cours d'action se développe dans le cadre de deux programmes articulés : un programme de recherche empirique d'anthropologie cognitive et un programme de recherche technologique dont la contribution au processus de projet a été systématisée par Theureau et Jeffroy (1994). Cette contribution porte plus particulièrement sur le passage de la description de l'activité aux recommandations du projet. Défini comme "comportement conscient ( $d u$ moins en partie), intentionnel, planifié, socialement contrôlé (ou dirigé) et significatif pour l'opérateur en situation de travail ", le cours de l'action est l'objet de descriptions détaillées qui permettent de formaliser des modèles d'analyse à des niveaux différenciés de l'organisation de l'activité (globaux ou locaux).

Lorsqu'ils sont associés au processus de conception, ces modèles plus ou moins généraux sont articulés au projet en développement en l'alimentant avec différentes informations à chaque étape. L'analyse globale du cours d'action est principalement développée dans les étapes initiales des projets, car elle permet de définir les fonctions et objectifs du système en conception (elle consiste à identifier et à caractériser les cours d'actions dans leur ensemble, ce qui pourrait être qualifié de macro-analyse).

Dans la phase de conception détaillée, l'analyse globale continue à fournir différentes contributions, mais cette analyse peut être approfondie par des analyses locales, réalisées sur l'ensemble du cours d'action ou ciblée sur des phases du cours d'action qui présentent un intérêt particulier pour la conception et que l'analyse globale n'a pas permis d'appréhender de manière satisfaisante. L'analyse locale est alors particulièrement intéressante pour caractériser les difficultés des usagers et pour identifier leurs origines en situation d'expérimentation sur une maquette ou un prototype.

D'autres difficultés méthodologiques doivent pourtant être surmontées pour passer de la description, globale ou locale, aux modèles opérationnels dans la perspective des concepteurs. Dans le cadre théorique du cours d'action, cette fonction est remplie par les hypothèses concernant la transformation du cours d'action qui associent les caractéristiques de l'organisation intrinsèque des cours d'action et les contraintes et effets extrinsèques (en relation avec l'état des acteurs, leur situation et leur culture) (Theureau, \& Jeffroy, 1994, p.106). Ces hypothèses possèdent un certain niveau de généralité qu'il faut encore particulariser avant de créer des règles utilisables dans le projet. Nous en arrivons ici au problème du passage entre l'analyse de l'activité et la formalisation de recommandations que 
nous avons identifié comme une question centrale dans toutes les approches de l'ergonomie de conception.

Néanmoins, et comme le reconnaissent les auteurs, si les hypothèses de transformation sont présentes dans les études de conception centrées sur le cours d'action, de rares modèles de transformation ont été produits, cette évaluation a été réaffirmée récemment: «Si de nombreuses hypothèses de transformations du cours d'action ont pu être produites, qui réalisaient une adéquation descriptive forte et débouchaient sur des propositions d'aménagement et de conception ergonomique dont on a pu juger de l'adéquation pratique, elles n'ont que rarement été organisées en modèles de transformations du cours de l'action et a fortiori en modèles de transformations du cours de l'action et de ses effets » (Theureau, 2004, pp. 239-240). La théorie du cours d'action étant encore en développement, les modèles de transformation ont été uniquement élaborés dans le cadre de recherches scientifiques et "doivent donc être vus plus comme des étapes à dépasser dans l'avenir que comme des points d'arrivée » (Ib. p. 240).

Par ailleurs, comme évoquée plus avant, l'approche du cours d'action interagit avec le processus de conception dans ses différentes étapes. D'une façon générale, ceci consiste à construire des recommandations pour le projet à partir d'analyses et de modélisations plus ou moins approfondies de l'activité de l'opérateur en débutant par les «orientations de conception » qui peuvent être produites par une analyse du cours d'action avant que le projet ne soit développé, en utilisant ici le savoir déjà accumulé dans la discipline ou lors d'interventions préalables. Outre les modèles et spécifications de projet, l'expérience accumulée permet alors de définir les conditions les plus favorables pour la production de ces spécifications durant un processus particulier de conception (la construction de modalités d'expérimentation sur une maquette, la constitution de groupes de suivi du projet, y compris les opérateurs).

Mais si dans un processus de conception, il existe différents modes de transfert de l'expérience et des connaissances accumulées par l'ergonomie, seul l'un d'eux fait l'objet d'un développement par l'approche du cours de l'action : "nous mettons ainsi l'accent sur un seul mode de transfert d'expérience des recherches et des études passées de conception centrée sur le cours d'action aux projets et processus nouveaux de conception, celui qui met en jeu des concepts et des méthodes explicites». Mais "la technologie ergonomique ne se réduit pas à cette technologie, et dans les processus de conception, l'expertise personnelle et les règles pratiques sont nécessaires » (Theureau, \& Jeffroy 1994, p.127, souligné par nous).

Progressivement, et dans la mesure des besoins des concepteurs, les orientations de projet sont spécifiées par le biais d'interactions des études du cours d'action avec le processus de conception en ayant soin de ne pas offrir plus de détails que nécessaire. Dans ce passage entre l'analyse de l'activité présente et la situation future, la notion de «situations caractéristiques", traduite dans les concepts du cours d'action, offre des bases de construction pour les spécifications plus détaillées. Il s'agit toutefois seulement du «premier saut» qui, actuellement, est encore restreint au champ de connaissance de l'existant placé côte à côte avec l'état du projet à chaque étape : l'analyse du cours d'action avance pas à pas avec le projet. Ces résultats doivent subir une transformation, une formalisation ultérieure, qui permet d'élaborer des règles de conception, plus ou moins générales, organisées ou non en système de règles.

«Le passage du diagnostic-pronostic à la contribution effective à la conception constitue un second saut de la connaissance de l'existant à la pratique de conception. Il ne s'agit jamais de recopier les situations précédemment analysées. C'est là que sont précisés les concepts directeurs et opérationnels pertinents et que sont définies les règles de conception, générales et ad hoc, organisées ou non en systèmes de règles (...). En définissant ces règles pour les différentes situations caractéristiques dégagées, on peut concevoir (...) des scénarios de conception et des modèles de conception. La notion de situation caractéristique permet d'apporter une nouvelle précision à ces derniers. Les scénarios de conception sont valables 
seulement pour des situations caractéristiques données. Ils sont particulièrement utiles lorsqu'on a pu dégager toute une série de situations caractéristiques fondamentalement différentes », (Theureau, \& Jeffroy, 1994, p. 128).

\section{5.- Configurations d'usage : récupérer l'expérience pour anticiper}

Dans la même lignée d'approche, nous avons également recherché «un modèle de conception, une représentation des cours d'action en situations futures, capables de guider la conception » qui puissent servir « de modèles d'analyse de ces cours d'action futurs». Ces modèles doivent posséder «un contenu plus riche et plus adapté aux données des cours d'action actuel ou futur ». Nous ne pensons pas non plus que ces modèles soient autosuffisants pour dispenser des analyses postérieures et des approfondissements au moment de la conception lorsque l'on sollicite l'expertise des ergonomes qui intègrent ou assistent l'équipe de projet.

Néanmoins, et comme les auteurs le reconnaissent, certaines questions restent en suspens pour opérer le passage de la description de l'activité en modèles de transformation. Quel niveau est le niveau de généralité/spécificité adapté à chaque étape du projet, surtout lorsque ces étapes ont lieu sur de longs intervalles de temps et concernent des acteurs de projet différents ? Parmi les règles de transformation des modèles qui génèrent des spécifications, quelles sont celles qui dépendent des connaissances accumulées et quelles sont celles utilisées pour les analyses réalisées au moment du projet ? Comment travailler, en pratique, la formalisation de recommandations qui évitent l'excès de généralités qui n'apportent rien de neuf aux concepteurs et l'excès de spécifications qui peut inhiber la création de nouveautés plus efficaces? Quels éléments minimaux doivent être contenus dans ces modèles de transformation et comment doivent-ils être articulés ?

Pour faire face au paradoxe de l'ergonomie de la conception (comment instruire un projet futur, encore non matérialisé, si les analyses ergonomiques doivent nécessairement avoir lieu dans des situations concrètes ?), les approches précédemment citées, on fait appel à l'analyse de situations de référence, mais le passage de l'analyse de l'activité ici et maintenant aux recommandations de projet reste une question en suspens. La formalisation basée sur le cours d'action se situe dans la même direction que celle des concepts d'analyse de situations de référence, $\mathrm{SAC}$ et $\mathrm{AAF}^{3}$ proposés par Daniellou (1988), mais privilégie les concepts et les modèles de formalisation par rapport à la construction sociale de l'intervention. Le problème de la traduction de l'analyse en spécifications de projet se complique lorsque les recommandations ne sont pas directement destinées au projet détaillé, comme exigé pour le projet de base des plateformes.

Entre la situation de référence et la situation future, il faut maintenant interposer les recommandations plus génériques, mais néanmoins effectives pour orienter le projet final. Comment éviter alors les recommandations excessivement génériques qui, pour l'essentiel, se limiteraient à dire que les situations futures doivent être ergonomiques, mais sans détailler la solution pour chaque projet, toujours singulier ? Comment s'assurer, en outre, que les recommandations issues des situations actuelles soient suivies dans le cadre des nouveaux projets si ceux-ci assument de nouveaux engagements et si le projet initial se transforme durant son cycle de vie, des études de base à l'exécution et, postérieurement, dans l'usage ? De façon générale, la stratégie adoptée par l'ergonomie est d'agir le plus tôt possible dans le processus de conception. Il s'agit de définir les conditions (connaissances de l'activité existante et des situations de travail) pour que cette intervention se fasse de la façon la plus 
pertinente. La notion de configuration d'usage cherche à avancer dans cette voie.

\section{1.- Identifier les configurations d'usage : comment élaborer des spécifications de projet basées sur l'activité}

Le principe général qui oriente la coopération entre l'ergonomie et l'ingénierie est la construction de spécifications de projet basées sur l'activité à partir d'une expérience forte d'expérience élaborée par la production et dans l'utilisation. Expérience, qui, une fois formalisée, alimenterait la fonction de projet. Néanmoins, du fait de sa nature située, historique et singulière, cette expérience se présente à la fois comme un moyen du projet et comme un problème: «congeler » l'expérience des opérateurs en spécifications semble trahir ce principe fondamental de l'ergonomie de l'activité et se rapproche dangereusement de la pratique des facteurs humains. Alors, comment transiter entre orientations sur le travail futur et les connaissances spécifiques des situations actuelles existantes ? À quel point, le certain et le possible déterminent-ils les situations qui auront effectivement lieu dans le futur?

Les spécifications ergonomiques, à tous les niveaux de généralité, seront efficaces dans la mesure où l'on ne crée pas de gênes définitives pour les futurs ergonomes qui devront détailler les nouvelles situations de travail en collaboration avec les concepteurs des installations. D'où notre option de formaliser les orientations issues de l'analyse de l'activité en situations réelles en termes de configurations d'usage. Si l'expérience est la base essentielle de l'analyse de l'activité, essentielle non seulement pour concevoir les systèmes technologiques, mais aussi pour les modifier et les perfectionner, il serait paradoxal de prétendre concevoir des systèmes ergonomiques sur la base de normes définitives et obligatoires, sans avoir recours à l'expérience de ceux qui les utilisent, et ceci de façon continue, car le projet est prolongé dans l'utilisation avec le développement de nouveaux schèmes d'action. Les configurations d'usage fonctionnent comme des contenus substantifs et des scénarios pour que les futurs concepteurs dialoguent avec les futurs usagers par le biais de l'expérience des usagers actuels. Elles prétendent inciter à la réflexion et à la construction de réponses à des questions précises du point de vue de l'activité : comment les opérateurs actionnent-ils cette vanne? Comment l'enlèvent-ils pour la maintenance? Comment cet instrument (manomètre) est-il inséré dans leur activité ? Les configurations d'usage alimentent la dialectique du projet et développent de façon permanente la conversation entre les concepteurs et l'usager par l'instrument et son activité, c'est-à-dire par l'activité instrumentale (Béguin, 1994 ; Rabardel, 1995). Il faut néanmoins qu'elles soient suffisamment proches de la réalité pour influencer le projet avant que ne soient définies les spécifications détaillées.

Nous pouvons nous inspirer de la proposition d'Alexander dans sa seconde phase (Alexander, 1977 ; 1981) lorsqu'il cherche à identifier ce qu'il avait nommé la « qualité sans nom » et le «mode intemporel de construire». Cet architecte qui abandonna son titre et s'auto-déclara «constructeur»- car il ne croyait plus en des projets a priori - comme à l'époque de la Synthèse de la Forme (Alexander 1966), chercha à identifier des configurations spatiales qui fonctionnent, de «bonnes formes » enregistrées dans chaque culture et incorporées dans l'expérience de chacun de nous, et qui peuvent orienter des conceptions de nouveaux espaces sans limiter la créativité ; "il existe d'infinies solutions pour un problème donné. Naturellement, il n'y a pas comment définir les détails de toutes les solutions en une seule proposition résumée. C'est toujours l'imagination créative du concepteur qui doit trouver une nouvelle solution au problème et qui soit adaptée à la situation spécifique» (Alexander, 1981, p. 206). Après avoir visité différents environnements construits de par le monde, Alexander identifia des normes de bonnes formes qui comportent au minimum trois ingrédients: des composantes de base, des relations spatiales entre elles et une action individuelle ou collective, développée dans une culture donnée. Une norme est l'unité entre des éléments spatiaux et constructifs, relations et 
activités, qui sont uniquement repérables par les émotions : "les normes qui proviennent de pensées et non pas de sentiments manquent totalement de réalité empirique (...) il y a une relation fondamentale entre l'équilibre d'un système de forces et nos émotions avec le respect de la norme engendrée par ces forces. Ceci a lieu parce que nos émotions abordent toujours la totalité de n'importe quel système » (Alexander, 1981, p. 224). C'est pour cette raison que le mode intemporel pour construire «ne peut être atteint : il se fait spontanément si nous le permettons » (idem p. 11). Dans sa forme plus générique, une norme est formulée sur la base de règles qui établissent les relations entre un contexte donné, un système de forces qui doit être identifié et une configuration qui permet à ces formes de se résoudre dans ce contexte. Bien qu'elles surgissent spontanément dans une culture donnée, il n'est pas aisé de découvrir ces configurations invariables de normes. Il est nécessaire «d'expliquer quelles sont les forces qui opèrent et (...) formuler la norme dans des termes qui l'accentue, car elle a contribué à résoudre un système de forces qui ne peut se résoudre sans elle » (idem p. 204).

De façon analogue, nous avons tenté d'abstraire de l'analyse de situations d'actions caractéristiques les configurations d'usage qui sont situées à mi-chemin entre les principes généraux de l'ergonomie, comme par exemple «faciliter l'accès à l'opérateur » et les détails de cet accès dans un projet donné. Mais contrairement à Alexander, qui s'arrête à la relation intuitive et atemporelle avec l'espace habité, nous pensons que l'analyse de l'activité permet d'avancer dans la description de ces configurations en formalisant partiellement les expériences négatives et positives des opérateurs concernés par le contact quotidien avec les installations, ce qui peut contribuer, en retour, au développement de projets plus adaptés. La «bonne forme" s'avère ainsi être un processus permanent d'aménagement entre les configurations d'usage, le projet détaillé et l'usage réel.

\section{2.- Configurations d'usage pour le projet du système de lancement de PIG}

L'opération et la maintenance du système de lancement et de réception de PIG sont considérées par les opérateurs des équipes de production et de maintenance des plateformes étudiées comme l'une des tâches les plus pénibles au regard de l'intensité des efforts pour actionner les vannes, de la criticité des postures et des déplacements nécessaires, comme l'on peut le constater dans l'explication ci-après. Avec la description détaillée de ce cas, nous essayons de démontrer comment l'analyse de l'activité, en associant évaluations subjectives et objectives, permet de caractériser des situations pénibles et d'identifier les éléments typiques d'une configuration d'usage capable de produire des recommandations utilisables en phases initiales du processus de conception.

Les PIG (Figure 1) sont des dispositifs qui se déplacent à l'intérieur des tubes. Ils sont impulsés par le fluide en propre et ont les finalités suivantes : inspecter et contrôler les tubes, ôter les liquides, prévenir la formation de dépôts organiques et inorganiques, faire le nettoyage interne et enlever les solides déposés. L'exploration de pétrole en eaux profondes justifie le perfectionnement de cette technologie (PIG) et son utilisation constante, spécialement pour ôter mécaniquement la paraffine formée, à cause des basses températures, à l'intérieur des tubes qui transportent le pétrole du puits jusqu'à la plateforme. 


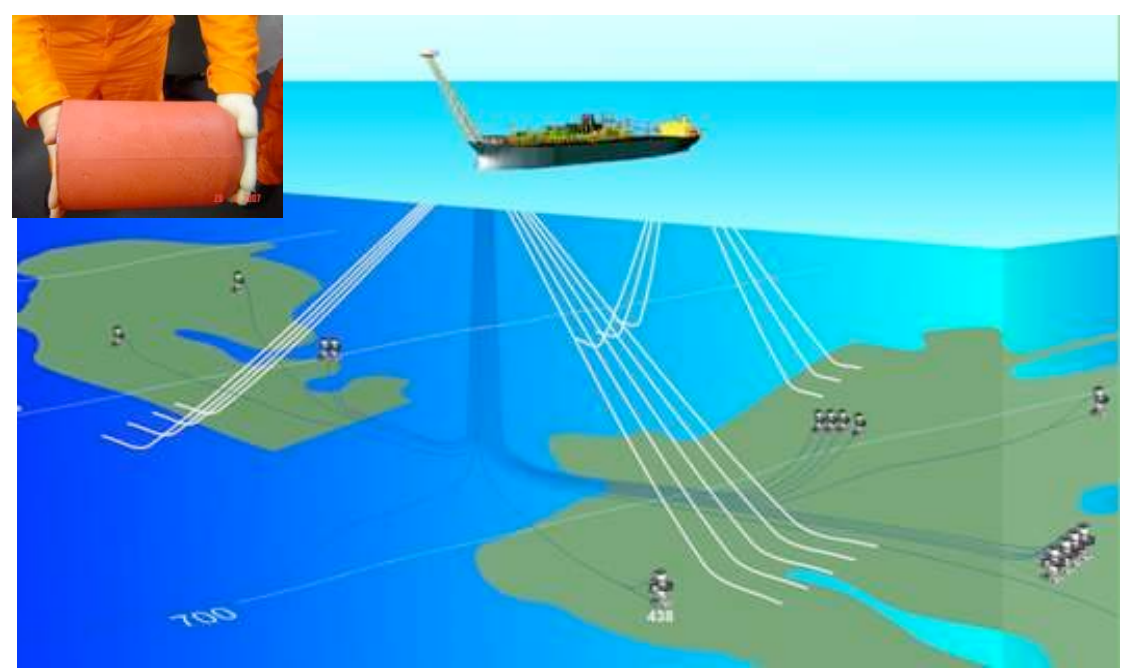

Figure 1 : Les PIG (petite photo) débouchent les tubes qui relient la plateforme aux puits de pétrole

Figure 1: The PIGs (small photo) open the tubes which connect the platform to the oil wells

Du point de vue méthodologique, l'essentiel de la contribution au projet des futures plateformes et à la construction des configurations d'usage est issu de l'analyse de l'activité, c'est-à-dire de l'observation et des verbalisations des opérateurs. Les verbalisations des opérateurs ont été décisives pour mettre en lumière les difficultés liées aux efforts et postures et les raisons des déplacements telles que : la rigidité des vannes manuelles, les nombreux déplacements pour l'ouverture des by-pass de la vanne de sécurité, les postures extrêmes pour contrôler la pression sur le manomètre.

Une condition particulièrement pénible est due aux déplacements fréquents, en utilisant des escaliers, réalisés par les opérateurs durant les manœuvres de lancement et de réception de PIG. Ces déplacements concernent entre autres l'accès aux by-pass des vannes de sécurité et la diminution de la pression (PSV). Ces by-pass sont situés avec les vannes à deux étages audessus du pont principal où se trouvent les dispositifs de lancement et de réception des PIG. Durant le projet, au regard de l'obligation du respect des règles de sécurité, le choix a été fait de regrouper toutes les PSV des différents systèmes au même endroit, au-dessus du pont principal. La surveillance de la pression interne des dispositifs de lancement et de réception est l'un des principaux aspects de l'activité. Les déplacements jusqu'au by-pass de la PSV sont réalisés pour contrôler si la pression du système est réellement à zéro. Bien que le manomètre de chaque station de LP/PR indique une pression basse ou proche de zéro, l'opérateur se déplace pour ouvrir le by-pass car il sait qu'il existe toujours une pression résiduelle qui a déjà été à l'origine d'accidents.

La configuration d'usage dans ce cas a été décrite de la forme suivante :

Dépressurisation de la chambre/by-pass de la PSV - manœuvre effectuée dans le but de viabiliser l'ouverture sans risque des chambres (aussi bien de lancement que de réception). Le gaz à l'intérieur de la chambre est libéré vers la torche (flare), par le by-pass de la PSV, par une action manuelle sur les vannes. Le gaz résiduel (à basse pression) est libéré par des soupapes présentes dans la chambre. La lecture du manomètre et l'observation de la sortie de gaz par la soupape sont des garanties pour l'opérateur qu'il peut ouvrir la chambre sans danger.

D'autres situations typiques pour actionner les systèmes PIG à l'origine des configurations d'usage ont concerné l'insertion et l'enlèvement des PIG et la fermeture du portillon (Figures 2 et 3). Comme nous le discuterons par la suite, le projet de ces systèmes peut 
favoriser la réalisation de ces activités.
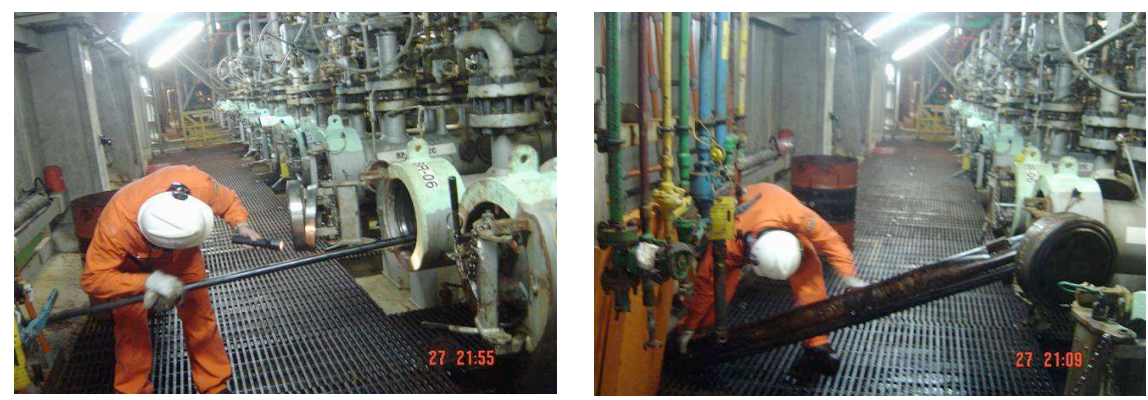

Figure 2 : Insertion et lancement du PIG - Source : Remiro, 2009

Figure 2: Inserting and launching the PIG - Source: Remiro, 2009
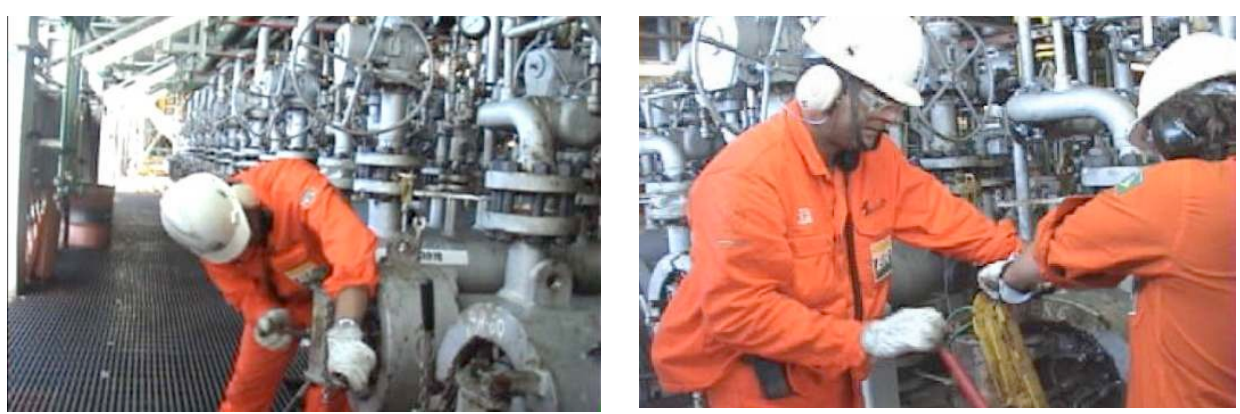

Figure 3 : Fermeture du portillon du système de PIG - Source : Remiro, 2009

Figure 3: Closing the PIG door system - Source: Remiro, 2009

Les configurations d'usage dans ces situations sont les suivantes :

- Insertion et dégagement du panier de réception - l'opérateur insère manuellement le panier de réception de PIG en évitant que la mousse soit déviée en direction des lignes de production, de la purge ou du by-pass à son arrivée. Après la réception, l'opérateur retire le panier pour enlever le PIG.

- Fermeture du couvercle de la chambre - fermeture et verrouillage du couvercle pour que la chambre puisse procéder à l'écoulement du gaz ou de l'huile internement.

Pour chaque configuration d'usage, il y a une association de conditions et de variables du projet susceptibles d'influencer les conditions de travail des opérateurs. C'est avec les configurations d'usage et les variables de projet que sont construites les recommandations techniques, donc les propositions pour le projet. Pour le système de lancement et de réception des PIG, 11 configurations d'usage ont été créées (8 sur la base de l'activité opérationnelle et 3 sur la base de l'activité de maintenance préventive) et 15 recommandations techniques (RT) y ont été associées.

Comme nous l'avons dit, les déplacements des opérateurs constituent une caractéristique marquante de l'activité de ce système. Ces déplacements sont épuisants et exigent de monter et descendre les étages de la plateforme par les escaliers. Cette constatation a été à l'origine de la recommandation suivante :

— «La localisation entre l'actionnement de la vanne de by-pass de la PSV proche et au même niveau que les chambres de lancement et de réception de PIG peut réduire le déplacement de l'opérateur durant l'exécution du lancement et de la réception du PIG. Il faut prendre en compte deux déplacements : l'un pour activer/désactiver le by-pass et l'autre pour prendre des informations sur le manomètre, qui est situé à côté de la chambre, mais dont l'information doit être relevée durant le by-pass ».

Pour les difficultés liées à l'insertion, le dégagement des PIG et la fermeture du portillon, on recommande : 
— « La zone devant le portillon des chambres doit être dégagée au maximum des conduites ou volants des vannes. Prévoir l'espace pour l'utilisation de dispositifs tels que bâtons et paniers de réception ».

— «Le dispositif de verrouillage du portillon doit permettre une fermeture rapide et demander peu d'efforts, tout comme le matériel et la structure du portillon doivent maintenir de bonnes conditions d'ajustage durant leur manipulation ».

En ce qui concerne la nécessité de suivre la mesure de pression à l'intérieur de la chambre, la recommandation suivante a été établie :

— «L'endroit du manomètre de la chambre devra faciliter la visualisation de l'opérateur quand il manipule la vanne du by-pass de la PSV. »

Sur la base du pourcentage de temps durant lequel l'opérateur et l'ouvrier de maintenance ont le bras au-dessus de la ligne du cœur, la recommandation suivante fut rédigée :

«Les vannes du système PIG doivent être positionnées dans des endroits d'accès facile et à des hauteurs favorables pour les opérateurs (en tenant compte de la norme anthropométrique de la population concernée par les opérations de ce système). Il est recommandé, de plus, que la détermination de la hauteur des vannes du système PIG tienne compte de la fréquence d'usage, du type et de l'effort réalisé pour leur manipulation ».

En termes de configurations d'usage, le projet des vannes du système PIG doit ainsi être orienté selon les recommandations traditionnelles des manuels de human factors mais reconsidérées dans un contexte défini par l'activité réelle de l'opérateur et de l'ouvrier de maintenance dont les principes conditionnent le projet sur les aspects suivants : 1) proximité des vannes ; 2) localisation sur un même niveau ; 3) position relative du manomètre ; 4) hauteur des vannes (surtout pour l'ouvrier de maintenance). Parmi tous ses principes, seule la hauteur est mentionnée dans les recommandations des manuels, car elle est visible même sur des modèles physiques de la relation corps/objets de travail. Les autres principes en lien avec la dynamique de l'activité n'y sont pas mis en évidence.

\section{3.- Les configurations d'usage : un regroupement d'aspects physiques, technologiques, d'orientations cognitives et de schèmes pratiques}

Pour développer les configurations d'usage, qui traduisent en forme positive les résultats d'analyse de l'activité validés par les travailleurs, les étapes traditionnelles de l'AET ont été suivies. Ces configurations ont été présentées et validées postérieurement par les concepteurs en réalisant une évaluation de ce qu'elles transmettaient des situations de travail envers les processus de conception dans la perspective de l'activité.

L'une des plus grandes difficultés rencontrées pour l'élaboration des recommandations techniques (RT) à partir des situations de référence a été d'extraire les configurations d'usage à partir des divers cas en évitant de s'attacher au cas d'une plateforme spécifique ou à des options adoptées sur d'autres plateformes visitées. Même lorsqu'une situation de référence proposait un bon exemple à copier, la recommandation assumait un ton plus général pour ne pas être associée au cas spécifique. Nous avons ainsi tenté d'éviter que la recommandation prenne un statut de norme obligatoire, au lieu de servir de référence, comme nous le prétendons, en laissant une marge de manœuvre pour que l'équipe du projet puisse innover et éventuellement améliorer l'état en la matière.

La notion de configuration d'usage permet de mieux comprendre comment les RT ont été construites et également comment elles doivent être utilisées. Ce qui définit une configuration d'usage est toujours le couplage entre, d'un côté, les aspects physicotechnologiques (environnement/espace, instrument/objet, équipement), le contexte social et les orientations cognitives (par exemple : ouvrir une vanne pour...) et, d'un autre côté, un schème pratique qui est sous-jacent à une activité donnée : (par exemple : comment vérifier 
la position du PIG dans la chambre fermée). Au sein d'une configuration d'usage, les éléments de la tâche, de l'environnement (de la situation) et de la propre activité sont articulés de façon pertinente pour orienter les décisions de projet depuis le début des études de base.

Dans les diverses analyses réalisées, nous avons tenté de mettre l'accent sur les relations (ou couplages) qui influencent le projet et certains aspects de l'activité qui ne comportent pas de conséquences immédiates pour une phase ou étape déterminée du projet et qui n'ont pas été prises en compte. En adoptant les configurations d'usage comme point de départ pour l'élaboration des recommandations et des solutions du projet, il a été possible d'abstraire des détails et des spécifications observés durant l'analyse ergonomique de l'activité qui n'ont pas besoin de figurer comme élément essentiel des configurations d'usage bien que ces dernières soient issues de ce matériel. À caractère général, les configurations d'usage ne décrivent pas toutes les variabilités et les spécificités de chaque plateforme, ni tous les détails des procédés et modes opératoires et ni les problèmes spécifiques mis en évidence par l'analyse ergonomique de l'activité.

Il ne s'agit pas d'une description empirique des relations spécifiques des situations de travail existantes, ni d'établir des concepts et modèles généraux de la relation sujet, objet et instruments, comme si une théorie générale permettait de générer des projets qui incorporent des modèles de l'activité future, ce qui est en contradiction avec la propre notion d'activité humaine comme action située, non déterminée a priori. Mais il s'agit d'alimenter le processus de projet avec des éléments de l'organisation de l'activité : schèmes pratiques, interactions cognitives, utilisations sociales des instruments et objets, y compris les configurations spatiales des situations de travail (objets, instruments et conditions matérielles). Ici les questions initiales peuvent être plus spécifiques : sur quels formalismes peut se fonder le passage de l'analyse de l'activité existante à la conception ? S'agit-il de descriptions copiées des technologies ou de constructions théoriques plus abstraites? Ces relations dérivent-elles des objets (ou schèmes d'utilisation cristallisés), de caractéristiques des situations ou du sujet ? Comment identifier et décrire ces éléments?

La contribution des configurations d'usage au processus de projet a été testée à différents moments, avant que le projet ne s'initie et au début de la phase détaillée de projet. À chaque moment son utilité pour les concepteurs a été démontrée, mais avec des appropriations différentes. Dans le cadre d'un travail ultérieur, les configurations du couplage ont été organisées en un «standard de zones » (zone patterns), présenté aux architectes pour définir le macro-projet du module d'installations de la plateforme (Conceição, 2011). Dans ce cas, les configurations ont été considérées pour discuter les directives du projet conceptuel (localisation des différents environnements des étages, proximité des environnements, surface maximum des environnements, etc.) et définir quelques caractéristiques du projet de base. Fonctionnant comme des objets intermédiaires, les configurations d'usage ont rendu les directives du projet plus concrètes en facilitant le développement de représentations partagées en ce qui concerne le futur milieu. Sans que nous nous y attendions, les concepteurs se sont réappropriés les configurations d'usage comme forme de représentations pour développer l'inter-compréhension avec le client au sujet des directives de projet (Conceição, 2011).

Les configurations d'usage ont également été testées dans des phases postérieures du processus de conception pour créer des alternatives pour les installations de production plus adaptées à l'activité. Il s'agissait ici d'intervenir au début de la phase de détail pour transformer des options déjà adoptées dans le projet de base. Même avec des marges de manœuvre très réduites, les configurations d'usage ont ainsi permis d'introduire dans le projet initial des améliorations plus adaptées à l'expérience des usagers, préalablement explicitée par les analyses des SAC, auxquelles, malheureusement, il n'a pas été possible de revenir vu les délais serrés pour critiquer les propositions et négocier des modifications 
(Oggionni, Duarte, \& Cordeiro, 2012).

Les configurations d'usage se sont montrées utiles en tant qu'instruments dans des méthodes rapides d'étude ergonomique pour répondre à une demande d'élaboration d'un programme d'ergonomie pour plateformes. Organisées en scénarios, il a été possible d'accélérer la collecte des demandes et la cartographie des situations critiques dans diverses unités, surtout pour les activités de maintenance qui ne peuvent pas toujours être observées directement. Elles ont ainsi fonctionné pour orienter les futures analyses détaillées, comme ce que l'on attend d'un ergonome qui participe à un projet.

Ces tests des configurations d'usage à différents moments du projet mènent à la conclusion générale suivante : les configurations d'usage instruisent directement le projet et outillent l'ergonome dans les phases initiales (projet conceptuel et de base); plus le projet avance, exigeant des analyses détaillées et des formalisations de modèles de l'activité, et plus l'usage des configurations d'usage sera indirect. Elles passeront au service de l'orientation pour l'ergonome qui intègre l'équipe du projet. Les configurations d'usage ne se présentent pas comme une check list mais comme un réservoir d'expériences cristallisées et de connaissances sur l'activité du travail durant les interventions ergonomiques où le temps d'accès aux SR en parallèle au projet est limité. Dans les gros projets, comme ceux des plateformes, il existe des déphasages temporels et des aléas qui ne facilitent pas la synchronisation entre les analyses détaillées des SAC et les demandes d'informations pour alimenter les différentes étapes de projet, qui ont toujours lieu avec de fortes restrictions des délais. Quelques mois de retard du départ, par exemple, peuvent impliquer une augmentation significative du coût global du projet. De grands équipements sont commandés avant même le projet de base (Duarte, Silva, Lima, \& Maia, 2010). Ici le projet consiste essentiellement à gérer les temporalités asynchrones qui consomment du temps et réduisent les marges de manœuvre de toutes les autres décisions du projet, y compris ergonomiques. Comme les autres approches qui font face au paradoxe de l'ergonomie de conception, les configurations d'usage demandent une restructuration de la conduite du projet pour créer un contrepoint aux pressions temporelles qui peuvent être contreproductives du point de vue du cycle de vie d'une plateforme et ouvrir la porte à une catalyse des expériences des divers acteurs par la confrontation de connaissances sur des situations de plus en plus fines.

Les configurations d'usage ont été validées par le fait que les concepteurs et les ingénieurs se sont appropriés ces formalisations, mais il est significatif qu'elles soient plus utiles quand les ergonomes les soutiennent tout au long du processus de conception. Ceci confirme l'équivoque des guidelines destinées directement aux ingénieurs et réaffirme la nécessité de la participation des ergonomes dans les équipes des projets.

Les projets comprennent également la structure organisationnelle, des procédés de gestion et de formation qui n'ont pas fait l'objet d'analyse dans le contexte de cette demande, tournée vers le projet des installations physiques des plateformes. Les résultats obtenus autorisent néanmoins à prendre en compte ces nouvelles cibles immatérielles qui, dans les demandes, actuellement, ont tendance à croître dans ce secteur avec le développement de projets de salles de contrôle à distance, de projets d'environnements collaboratifs, de transfert d'activités offshore vers le milieu onshore et d'autres initiatives d'opérations intégrées.

\section{6.- Conclusion : de l'analyse de l'activité aux modèles pour le projet}

Le souci de fournir des éléments aux ingénieurs de projet est partagé par l'ergonomie des facteurs humains et par l'ergonomie de l'activité. Toutefois, en fournissant des orientations et des paramètres de projet isolés des situations réelles de travail, ce qui est gagné en détail de précision technique est perdu en pertinence pratique pour l'opérateur. Les analyses de situations de référence sur les plateformes pétrolières ont permis l'identification de 
difficultés, comme dans le cas du système PIG, qui peuvent être évitées en cas d'anticipation de certaines configurations d'usage à partir de l'étude de base, ce qui permet à l'ergonomie d'agir effectivement depuis le début du projet.

Il ne faut toutefois pas confondre ces recommandations avec les spécifications ergonomiques issues de l'analyse de l'activité en situation de référence, directement utilisable comme situations futures probables (Daniellou, $1988 ; 1992$ ), ou un tant soit peu avec les guidelines typiques des manuels de human factors et les normes techniques qui ont des valeurs prescriptives. L'identification des configurations d'usage pour instruire le projet de base ne remplace pas non plus les futurs ergonomes qui agiront dans l'équipe du projet, conjointement aux ingénieurs et concepteurs, ou la construction technique et sociale nécessaire dans n'importe quelle intervention ergonomique. Elles créent seulement des orientations préliminaires, relativement générales, pour que le travail des futurs usagers soit pris en compte depuis le départ du projet de base et que, de cette forme, soient anticipés les problèmes, comme dans le cas décrit par exemple les efforts et les postures non adaptées, les difficultés d'accès et les déplacements, la difficulté d'exécution (ou d'opérationnalité/ fonctionnalité), les risques d'accident, le besoin d'augmenter le nombre d'opérateurs concernés par l'exécution de la tâche, etc.

Ces recommandations sont d'autant plus nécessaires dans le cas de l'organisation de projets qui comme ceux d'une plateforme comprennent des dizaines de fournisseurs d'équipements divers. Avec l'accroissement de la sous-traitance du projet, il est en effet nécessaire, pour orienter et contrôler le résultat final, que le client fournisse des recommandations qui se matérialisent sous la forme de plans avec des conditions adaptées d'opération et de maintenance. C'est également le cas pour des systèmes de haute technologie, fabriqués par des fournisseurs spécialisés (vannes, motobombes, turbogénérateurs, etc.) qui ont tendance à négliger les aspects qui ne sont pas spécifiquement techniques, comme c'est le cas des spécifications ergonomiques. Avec l'identification des configurations d'usage, les spécifications et la mémoire descriptive du projet commandées aux fournisseurs seront enrichies et les décisions en amont intègreront une marge de manœuvre plus large afin que les concepteurs tiennent compte de l'activité réelle au moment du projet détaillé.

Les défis pour l'élaboration de ces spécifications techniques sous forme de configurations d'usage sont nombreux. Et diverses questions se posent, particulièrement au sujet des relations entre la nature statique et figée des spécifications et la nature dynamique et créative du projet. Comment s'assurer que les recommandations issues des situations actuelles soient suivies dans le cadre de nouveaux projets, si ces derniers assument de nouveaux engagements et si le projet initial se transforme durant son cycle de vie, des études de base à l'exécution et, postérieurement, en utilisation?

Les descriptions antérieures de configurations d'usage proposent une base empirique pour confronter cette notion à d'autres notions similaires dans le domaine de l'ergonomie de l'activité, propositions brièvement présentées plus avant: activité future probable, activité instrumentale et cours d'action. Ces approches opérationnalisent le passage de la description de l'activité en situations existantes pour alimenter le processus de conception de nouvelles situations par les notions suivantes: «SAC», «instrument», et «modèle de transformation ». Wisner avait déjà proposé auparavant la notion de «modèle opérant » qui peut être considérée comme la première formulation de l'ergonomie de l'activité pour traduire les descriptions systématiques de l'activité de travail, alors appelée «travail réel », en orientations de projet.

Face au nouveau continent qui se profilait par la reconnaissance de la distance entre le travail prescrit et le travail réel, la notion de modèle opérant cherchait alors à produire un savoir sur l'activité dans l'objectif de transformer les situations de travail. Dans la définition fournie par Wisner en 1972, les conditions nécessaires afin que les modèles soient opérants étaient établies: représenter les aspects essentiels du réel, c'est-à-dire «avoir une validité 
descriptive des conséquences pour l'opérateur et être capable d'aboutir à des solutions efficaces » (Béguin 2010, p. 19). Bien que synthétiques, nous avons retrouvé, au sein de cette définition, les éléments fondamentaux des recommandations ergonomiques reconnaître les aspects essentiels dans la situation de travail et élaborer des spécifications de projet qui contribuent à des solutions plus efficaces du point de vue de la production et de la santé. Proposition dont le développement postérieur par d'autres approches s'est retrouvé face à des questions également fondamentales : comment décrire l'activité développée sous le travail réel ? Quels sont les éléments essentiels dans cette description ? Comment passer de la description de l'activité, ici et maintenant, à des recommandations de projet vraiment efficaces en sachant que le projet cible une situation future ?

Dans une vision plus générale, Béguin (2010) propose une clé de lecture de ces diverses approches par la voie de la catégorisation des trois orientations de prise en compte de l'activité dans la conception : cristallisation, plasticité et développement. Sur la base de ces catégorisations, nous pouvons maintenant reprendre les notions par lesquelles les approches de l'ergonomie de la conception tentent d'opérationnaliser ses propres «modèles opérants » pour répondre aux demandes de projets centrés sur l'activité et montrer comparativement où la notion de configurations d'usage y trouve une place.

La description des configurations d'usage présentées par le système de lancement des PIG permet de clarifier les principales différences et points communs avec les notions de SAC et de schème. Par rapport aux SAC, la configuration d'usage comprend plus d'un élément qui nous parait essentiel pour avancer dans la capitalisation de l'expérience : modes opératoires plus ou moins généraux (ou certains des éléments considérés essentiels), soulignés par l'analyse de l'activité en situation de référence. Une description minimale des configurations d'usage exige ainsi d'évoquer les opérateurs (sujets) + les objets de leur action + l'intention poursuivie + les modes opératoires + les éléments contextuels. Comparativement, les configurations d'usage s'approchent ainsi encore plus de l'activité, en plaçant les modes opératoires comme un élément de production du couplage avec les autres constituants. Dans cet aspect, elles sont plus proches des modèles du cours d'action, qui sont orientés par la «priorité de l'intrinsèque ». En fonction de leur orientation dans les phases initiales de projet, et éventuellement sans accès aux SR à ces moments-là, les configurations d'usage assument volontairement un niveau moyen de formalisation. Elles sont plus restreintes que les SAC qui abordent un large éventail du général au singulier.

Pour l'approche instrumentale, les éléments valorisés sont inversés: le modèle SAI Situation d'Activité Instrumentée (Rabardel, 1995, p. 66), malgré l'utilisation du terme «situation », privilégie les liens entre sujet, instrument et objet, dans le contexte duquel l'élément principal, pour agir dans le projet, est le schème qui détient l'essentiel des expériences d'usage. Comme le processus de genèse instrumentale constitue un moment fort de cette approche, toute anticipation dans la phase de projet est limitée par le développement postérieur de l'activité. Dans le contexte de cette approche, l'environnement est plutôt considéré comme un facteur externe qu'un élément constitutif de l'activité.

L'approche du cours d'action propose d'intégrer tous ces éléments, sur la base d'une description rigoureuse de l'activité qui, par la suite, doit être traduite en modèles de conception. Ceci constitue toutefois le point le moins développé de cette théorie (Theureau, \& Jeffroy, 1994, p. 107). La notion de configuration d'usage peut contribuer à cette formalisation bien qu'elle ne fasse pas appel au même cadre conceptuel.

Avec en toile de fond les contributions antérieures, les recommandations formulées à partir des configurations d'usage identifient ainsi quelques pistes pour faire face au problème de la capitalisation de l'expérience et sa formalisation en termes utilisables par les concepteurs, ergonomes et autres acteurs :

- L'association entre éléments de l'activité et de la situation, -l'activité conformant un système matériel - ; le modèle de conception incorpore des relations subjectives liées à 
l'activité de l'opérateur avec des aspects organisationnels et des propriétés des systèmes techniques ;

- L'explication du pourquoi de la recommandation permet au concepteur de s'approprier la règle de projet et de devenir créateur de spécifications ergonomiques dans le contexte du projet, même en cas de changement d'options techniques.

— L'organisation en systèmes de règles, y compris les règles particulières et spécifiques, à différents niveaux d'abstraction, en mettant l'accent sur les arrangements intermédiaires qui concrétisent les principes généraux, mais sans détailler de solution spécifique ;

— La systématisation de l'expérience, qui facilite la participation d'ergonomes et d'opérateurs au processus de projet et augmente la possibilité d'anticipation avec, d'ailleurs, une économie de temps dans la définition des SAC et des analyses ;

— La formalisation des recommandations intermédiaires, à mi-chemin entre les directives de projet (orientations de projet), concepts et principes génériques et spécifications détaillées de projet, immédiatement utilisables par les concepteurs, mais en anticipant les phases du projet où les règles et les modèles intermédiaires seront nécessaires. C'est normalement le rôle de l'ergonome ou des opérateurs participant au projet de contribuer directement à ces spécifications par le biais de leur expérience professionnelle.

Pour que les règles/modèles de projet soient utilisables et utilisés par les concepteurs, la forme de présentation et l'organisation doivent assumer la perspective du concepteur et être axées plutôt sur les objets de son activité que sur les descriptions de l'activité des usagers. Du point de vue méthodologique, la formulation et le raffinement des configurations d'usage sont passés par la validation des concepteurs (ce qui n'est pas encore la validation par la pratique des concepteurs en situation de projet), ce qui a permis de progresser vers l'intégration des «contraintes de la conception» (voir Theureau \& Jeffroy, 1994, p. 108). Cet effort d'abstraction/ spécification a conduit à une étroite articulation entre les directives générales de projet et les solutions spécifiques, évitant ainsi le risque d'obsolescence précoce des recommandations en cas de modifications des normes technologiques, ou de changements paradigmatiques liés aux choix stratégiques, tout en maintenant le « duo» activité/technologie. Actuellement, elles sont utilisées pour orienter le projet des unités de production présalifère, la nouvelle frontière de l'exploration des réserves brésiliennes qui exigent des solutions innovantes dans le cadre de la conception générale des plateformes et des composantes spécifiques. Mais ceci est une autre histoire...

\section{BIBLIOGRAPHIE}

Alexander, C. (1966). Notes on the synthesis of the form. Cambridge: Harvard UP.

Alexander, C. (1977). A pattern language. New York: Oxford UP.

Alexander, C. (1981). El modo intemporal de construir. Barcelona: Gustavo Gili.

Attwood, D.E., Deeb, J.M., \& Danz Reece, M.E. (2004). Ergonomic Solutions for the Process Industries, USA, Elsevier.

Béguin, P. (1994). De l'individuel au collectif dans les activités avec instruments. Thèse de doctorat d'Ergonomie, Paris: Laboratoire d'Ergonomie du CNAM.

Béguin, P. (2010). Conduite de projet et fabrication collective du travail : une approche développementale. Thèse d'Habilitation à Diriger des Recherches - HDR, Université Bordeaux 2.

Béguin P., \& Weill-Fassina, A. (Eds.). (1997). La simulation en ergonomie : connaître, agir et interagir. Toulouse: Octarès.

Carballeda, G. (1997). La contribution des ergonomes à l'analyse et à la transformation de l'organisation du travail : l'exemple d'une intervention dans une industrie de process continu. Thèse de doctorat d'Ergonomie. Paris: Laboratoire d'Ergonomie du CNAM.

Chapanis, A. (1996), Human Factors in Systems Engineering. John Wiley \& Sons.

Chengalur, S.N., Rodgers, S., \& Bernard, Th. (2004). Kodak's Ergonomic Design for people at work. New Jersey, 2a Edição: John Wiley \& Sons. 
Clot, Y. (1998). Le travail sans l'homme? Pour une psychologie des milieux de travail et de vie. Paris: La Découverte.

Conceição, C. (2011). Do uso para o projeto : a transferência de experiência operacional para a concepção de espaços de trabalho em plataformas offshore. Thèse de doctorat, Programa de Engenharia de Produção - Université Fédérale de Rio de Janeiro.

Daniellou, F. (1988). Ergonomie et démarche de conception dans les industries de process continus, quelques étapes clefs. Le Travail Humain, 51(2), 184-194.

Daniellou, F. (1992). Le statut de la pratique et des connaissances dans l'intervention ergonomique de conception. Thèse d'Habilitation à Diriger des Recherches - HDR. Université de Toulouse.

Daniellou, F. (2004). L'ergonomie dans la conduite de projets de conception de systèmes de travail. In P. Falzon (Ed.), Ergonomie (pp. 359-374). Paris : PUF PP.

De Keyser, V., De Cortis, F., \& Van Daele, A. (1988) The approach of Francophone Ergonomy: studying New Technologies. In V. De Keyser, T. Quale, B. Wilpert, \& S. A. Ruiz Quintilla (Eds.), The meaning of work and Technological options (pp. 147-163). London: John Willey \& Sons.

Du Roy, O., Hunault, J.-C., \& Tubiana, J. (1985). Réussir l'investissement productif. Paris: Éditions d'Organisation.

Duarte, F. (1994). A Análise Ergonômica do Trabalho e a Determinação de Efetivos, Thèse de doctorat, Programa de Engenharia de Produção - Université Fédérale de Rio de Janeiro.

Duarte F. (Ed.), (2002). Ergonomia e Projetos nas Indústrias de Processo Contínuo, Rio de Janeiro: Editora Lucerna.

Duarte, F., Silva G., Lima, F., \& Maia, N. (2010). Ergonomics Guidelines for the Design Process, Society Petroleum Engineers - SPE International Conference on Health, Safety and Environment, Rio de Janeiro.

Garrigou, A., Daniellou, F., Carballeda, G., \& Ruaud, S. (1995). Activity Analysis in Participatory Design and Analysis of Participatory Design Activity. International Journal of Industrial Ergonomics, 15, 311-327.

Grandjean, E. (1998), Manual de Ergonomia, Artes Médicas, Porto Alegre, Brasil.

Haslegrave, C., \& Holmes, K. (1994). Integrating ergonomics and engineering in the technical process. Applied Ergonomics, 25(4), 211-220.

Hendrick, H.W., \& Kleiner, B. ( 2002). Macroergonomics: Theory, Methods, and Applications. New Jersey: Taylor \& Francis,

Iida, I. (1990), Ergonomia : projeto e produção. São Paulo: Editora Edgard Blücher.

Jeffroy, F. (1987). Maitrise de l'exploitation d'un système micro-informatique par des utilisateurs non-informaticiens - analyse ergonomique et processus cognitif. Thèse de Doctorat d'Ergonomie, Université Paris XIII.

Jeffroy, F. (1992). Les recommandations en ergonomie. Paris: Génie Logiciel et Systèmes Experts, No $29,40-46$.

Laville, A., \& Teiger, C. (1972). Nature et variations de l'activité mentale dans les tâches répétitives : essai d'évaluation de la tâche de travail. Le Travail Humain, 35(1-2), 99-116.

Klein, G.A., \& Brezovic, C.P. (1986). Design engineering and design process: decision strategies and human factors literature. In Proceedings of Human Factors Society 30 th Annual Meeting (pp. 771775).

Maline, J. (1994). Simuler le travail. Paris: Éditions de l'ANACT.

Meister, G. A., \& Farr, D.E. (1967). The Utilization of Human Factors Information by Designers. Human Factors, 9, 71-87.

Oggionni, B., Duarte, F., \& Cordeiro, C. (2012), Ergonomics in projects of oil platforms in a change context. 18th IEA Congress, Work: A journal of Prevention, Assessment and Rehabilitation, Vol 41, Supplement 1/2012, pp. 107-113.

Ombredane, A., \& Faverge, J.M. (1955). L'analyse du travail, Paris: PUF.

Pagenhart ,A., \& Buset, H.; (1998). Experience transfer from operational Environments to instalation Design: Why, How and What. Caracas: Conferência Internacional - Saúde, Segurança e Meio Ambiente, SPE - Society of Petroleum Engineers. 
Rabardel, P. (1995). Les hommes et les technologies. Approche cognitive des instruments contemporains. Paris: Armand Colin.

Remiro, R.R. L, (2009). Determinantes da carga física de trabalho em plataformas de petróleo: o caso da operação de PIG. Dissertação de Mestrado, Programa de Engenharia de Produção $\mathrm{PEP} / \mathrm{COPPE} / \mathrm{UFRJ}$

Salvendy, G. (1982). Handbook of human factors. New York: John Wiley \& Sons.

Simon, H. A. (1996). The Sciences of the Artificial. USA: $3^{\text {a }}$ Edição, MIT Press.

Swirenga, S.J., Morton, K., \& Boff, K.R. (1990). Issues concerning the use of human engineering information: the system designers' perspective. In IEEE International Conference on Systems Engineering, pp 881-885.

Theureau, J. (2004). Le Cours d'Action : Méthode Élémentaire, 2e édition. Toulouse: Octarès Éditions.

Theureau, J. (2009). Le Cours d'Action : Méthode Réfléchie, Toulouse: Octarès Éditions.

Theureau, J., \& Jeffroy, F. (Org) (1994). Ergonomie des situations informatisées. Toulouse: Octarès Éditions.

Theureau, J., \& Pinsky, L. (1984). Paradoxe de l'ergonomie de conception et logiciel informatique. Paris: Revue des Conditions de Travail, 9.

Wisner, A. (1972). Le diagnostic en Ergonomie ou le choix des modèles opérants en situation réelle de travail, Coll Ergonomie et Neurophysiologie du travail No 28, Paris: CNAM.

Wisner, A. (1987). Por dentro do trabalho, Ergonomia: método e técnica. São Paulo: FTD Oboré,

Wisner, A (1994). La cognition et l'action située : conséquences pour l'AET et l'Anthropotechnologie. Toronto, Actes du Congrès de l'IEA.

Wulff, I.A., Westgaard, R.H., \& Rasmussen, B. (1999a). Ergonomic Criteria in large-scale engineering design - I Management by document only? Formal Organization VS. designers perceptions. Applied Ergonomics, 30, 191-205,

Wulff, I.A., Westgaard, R.H., \& Rasmussen, B. (1999b). Ergonomic Criteria in large-scale engineering design - II Evaluating and applying requirements in the real world design. Applied Ergonomics, 30, 207-221.

Zarifian, P. (1995). Le Travail et l'événement. Paris: Harmattan

\section{RESUME}

L'intervention des ergonomes dans les étapes initiales d'un projet de conception dépend de la capacité d'anticipation des caractéristiques de l'activité future des situations de travail en transformation. Ce paradoxe de l'ergonomie de conception se voit « traité » par différentes approches et divers modèles conceptuels. Ainsi, les Situations d'Actions Caractéristiques (SAC), les schèmes d'utilisation et les propositions de transformation basées sur le cours d'action visent à offrir aux concepteurs des informations sur l'utilisation des systèmes techniques. Toutes ces approches instruisent le passage entre l'analyse du travail en situation réelle et les besoins du projet. C'est dans le même esprit que nous introduisons dans cet article la notion de configuration d'usage. Nous y présentons des exemples de ce concept pour orienter le projet de conception de futures plateformes de pétrole, et ce depuis la phase des études initiales. Nous prétendons en effet développer des configurations qui soient efficaces afin de transmettre les expériences accumulées dans le domaine des plateformes et pour transférer, dans le cadre de projets futurs, des conditions d'exercice du travail adaptées au déroulement de l'activité. Outre la contribution des expertises propres aux opérateurs, ergonomes et concepteurs, l'objectif est de créer une base de connaissances organisée en termes de configurations d'usage qui favorise le 
transfert d'expérience, et ce avant même la constitution des équipes du projet.

\section{MOTS-CLES}

retour d'expérience, projet centré sur l'activité, recommandations ergonomiques, configurations d'usage

\section{REFERENCEMENT :}

Duarte, F, \& Lima, F. (2012). Anticiper l'activité par les configurations d'usage : proposition méthodologique pour conduite de projet. Activités, 9(2), 22-47, http://www.activites.org/v9n2/v9n2.pdf

Acticle soumis le 28 juin 2011, accepté pour publication le 29 juillet 2012 